\title{
Boundary Detection in Piecewise Homogeneous Textured Images
}

\author{
Stefano Casadei ${ }^{1,2}$, Sanjoy Mitter ${ }^{1,2}$ and Pietro Perona ${ }^{3,4}$ \\ 1 Massachusetts Institute of Technology 35-308, Cambridge MA 02139, USA \\ e-mail: casadei@lids.mit.edu \\ 2 Scuola Normale Superiore, Pisa, Italy \\ ${ }^{3}$ California Institute of Technology 116-81, Pasadena CA 91125, USA \\ 4 Università di Padova, Italy
}

\begin{abstract}
We address the problem of scale selection in texture analysis. Two different scale parameters, feature scale and statistical scale, are defined. Statistical scale is the size of the regions used to compute averages. We define the class of homogeneous random functions as a model of texture. A dishomogeneity function is defined and we prove that it has useful asymptotic properties in the limit of infinite statistical scale. We describe an algorithm for image partitioning which has performed well on piecewise homogeneous synthetic images. This algorithm is embedded in a redundant pyramid and does not require any ad-hoc information. It selects the optimal statistical scale at each location in the image.
\end{abstract}

\section{Introduction}

The problem of texture analysis (recognition and segmentation) has traditionally been approached by computing locally defined vector-valued "descriptors" of each region in the image. The texture recognition problem is thus reduced to a conventional classification problem, and boundary detection may be performed by locating areas of rapid change in the descriptor vectors, or, dually, by clustering regions with similar descriptors. Constraints on texture analysis algorithms come from the physics and the geometry of image formation: often one would like to ensure invariance with respect to changes in illumination, scaling and rotation, sometimes also to tilt and slant.

The search for good local descriptors has been the focus of much work with two main classes of descriptors being favored in the more recent literature: (a) linear filters followed by elementary nonlinearities and smoothing (e.g. [1, 2, 3, 4]), and (b) different statistics of brightness computed on image patches (e.g. $[5,6,7])$. The filtering framework has natural characteristics for addressing the scale- and rotation-invariance issues: if each filter category is present at multiple scales and orientations, and if the discretization is fine enough, the representation of image properties given by the filter outputs is roughly scale and rotation-invariant.

A number of important issues having to do with scale selection and response normalization have remained virtually unanswered: What are the basic regularity hypotheses that a texture has to satisfy for the local descriptor approach to work? How does one identify automatically the proper scale for analyzing a texture, and how does one choose the thresholds for declaring a boundary?

In this paper we make explicit and formalize a general assumption about texture regularity. We show how this assumption can be used to find texture boundaries and we

\footnotetext{
* Research supported by Airforce grant AFOSR-89-0276-C and ARO grant DAAL03-86-K-0171, Center for Intelligent Control Systems
} 
discuss its relation with the scale selection problem. We observe that two independent scale parameters exist and must be dealt with. Finally, we show some results of an efficient redundant-pyramid algorithm for computing homogeneous texture regions. This algorithm is (approximately) translation-, scale- and illumination invariant.

\subsection{Overview of the contents}

A general assumption that forms the basis of all approaches to texture analysis is that a texture at some level of description is homogeneous. Although this is not a new idea, a precise definition of what this means is still missing. The difficulty in defining texture homogeneity is rooted into the two conflicting natures of texture, namely, randomness and regularity. A specific texture may be modeled as a realization of a random function. We propose to define a random function as homogeneous if, with probability one, spatial averages of any local operator are constant with respect to space in the limit of infinite size of the averaging region. To quote Wilson $[8,9]$, when the scale of averaging is infinite the class-localization of the texture pattern must become infinitely accurate. Of course, in practical circumstances we cannot take averages over infinite regions: for practical purposes the averaged texture features (i.e. the averaged output of the local operators) must be close to space-independence for finite averaging regions. When this happens we say that we are near the thermodynamic limit of a given texture. Due to the trade-off which exists between reliably estimating texture properties (which requires large averaging windows) and localizing the textured regions (which can be done more accurately if the data are not too blurry) it is important to be able to estimate the smallest averaging scale that approaches the thermodynamic limit.

The scale of averaging will also be called statistical scale or external scale. It must be distinguished from a different scale parameter: the feature scale or internal scale. The latter corresponds to the size of the support of a given local operator. These two scale parameters are unrelated (apart from the obvious fact that the external scale must be greater than internal scale). This is illustrated in figure 1. Note that even if the scale of the texture elements is the same for all images, the behavior along the statistical scale is quite different. A multiscale representation of a textured image should therefore contain two scale parameters (this observation has been independently made by Eric Saund, personal communication).

In order to select the most appropriate external scale(s) for analyzing a given texture we need to quantify the level of variability of the descriptors at any given statistical scale. To this purpose we define a real positive function, the dishomogeneity function, of two arguments, spatial position and statistical scale. Low dishomogeneity values will indicate that the thermodynamical limit has been reached. In order to be useful in texture analysis such a dishomogeneity function must satisfy two elementary properties:

1. The dishomogeneity of a region which contains only one type of texture is zero.

2. The dishomogeneity of a region which contains a texture boundary is strictly positive if the set of local descriptors is rich enough.

In section 2 we state two results (Theorems 1,2 ) which ensure that the dishomogeneity function satisfies these two properties in the thermodynamic limit for piecewise homogeneous textures.

Figure 2 demonstrates these concepts on a finite image. Note how the dishomogeneity of a given texture approaches 0 when the statistical scale is sufficiently large (left). However when the analyzed region goes over a texture boundary the dishomogeneity 

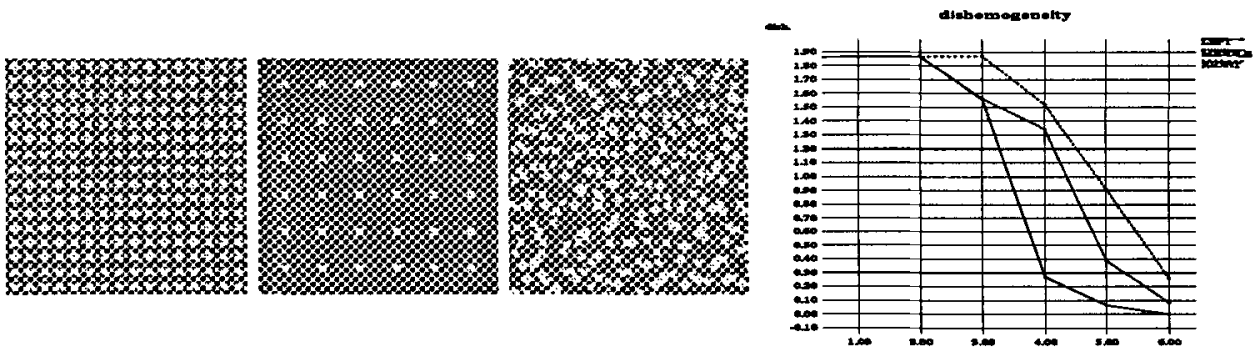

Fig. 1. Averages over larger regions are required to detect the regularity of the pattern in textures which are sparser or contain more "randomness" since the dishomogeneity of these textures approaches zero at larger statistical scales than dense periodic textures. At right the dishomogeneity function, maximized over the image, is plotted vs. statistical scale $k$ (i.e. $\max _{x} g^{\prime}\left(2^{k}, x\right)$ vs. $k$, see section 2.5 ) for the three different textures. Note that the dishomogeneity value of e.g. 0.3 is attained approximately at $k=4, k=5$ and $k=6$ for the left, center and right textures respectively. This suggests that a multiscale representation of the image should treat feature scale and statistical scale as independent dimensions of analysis.
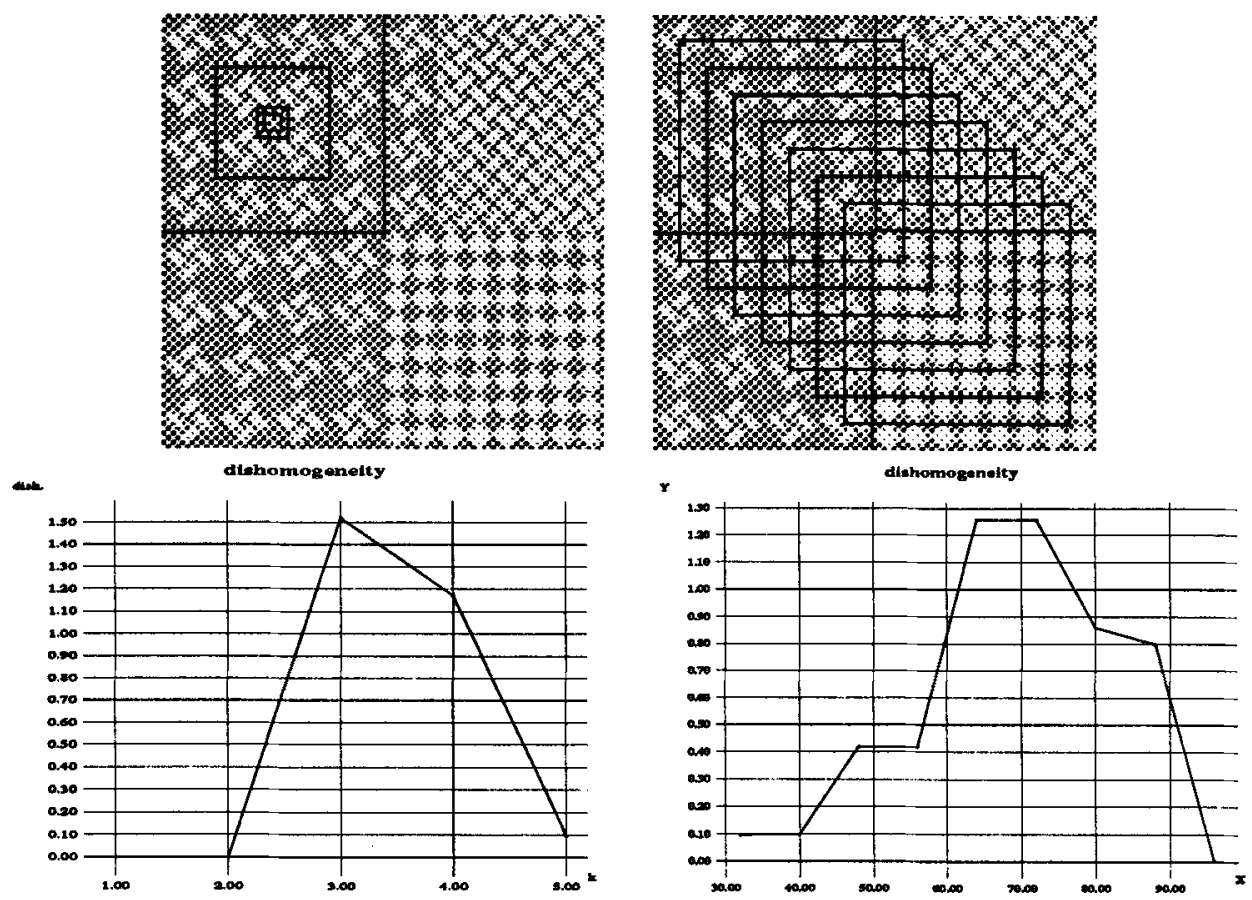

Fig. 2. The dishomogeneity at different scales and positions. The dishomogeneity inside each square region shown in the upper figure is plotted below. Left: the dishomogeneity of a given type of texture as a function of statistical scale is shown for a constant localtion in the image. The $\mathrm{x}$-coordinate, $k$, is the logarithm of the statistical scale. The linear size (length of the side in pixels) of each square is $2^{k+1}(k=2,3,4,5)$. The dishomogeneity at fine $(k=1,2)$ scales is zero because at the considered location only the constant backgound colour is present in the smaller squares. Right: the dishomogeneity at several positions in the image. The $\mathrm{x}$-coordinate now represents position in the image; the scale is held constant at $k=5$. Note that dishomogeneity is high when the region contains a texture boundary. 
becomes suddenly larger (right). Figure 3 shows how the dishomogeneity function is constructed.

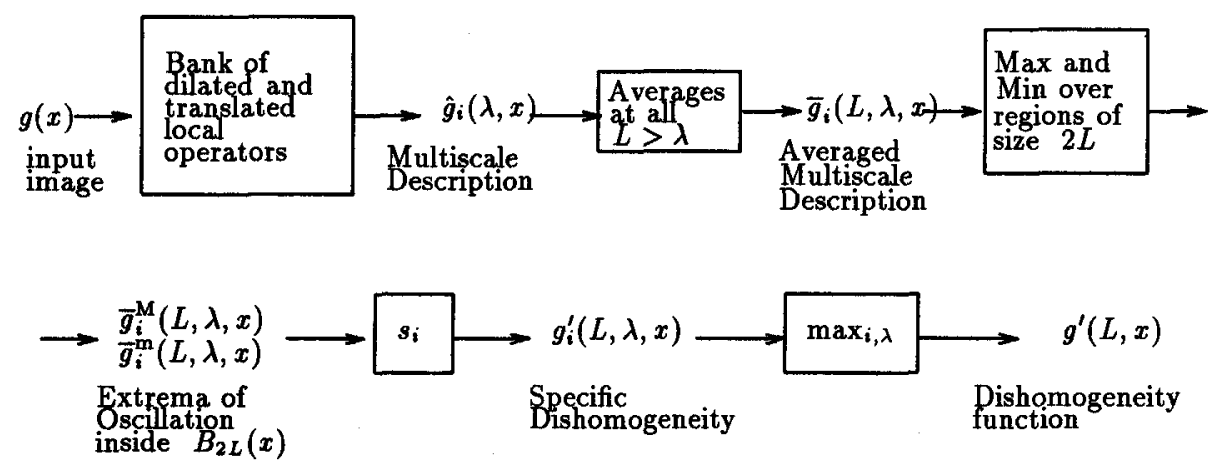

Fig. 3. Computation of the dishomogeneity function $g^{\prime}(L, x)$.

Our segmentation algorithm selects the optimal statistical scale at each location in the image by minimizing the dishomogeneity function (plus a penalty against small scales) among all possible scales.

\section{Multiscale Representations for textured images.}

\subsection{Notation.}

Let us introduce some notation. An image $g$ will be for us a real function $g: \mathbf{R}^{2} \rightarrow \mathbf{R}$. The set of all images is $G$. For $x \in \mathbf{R}^{2}$ let $T_{x}: G \rightarrow G$ be the translation operator defined as $\left(T_{x} g\right)\left(x^{\prime}\right)=g\left(x^{\prime}-x\right)$. Similarly, for $\lambda>0, D_{\lambda}: G \rightarrow G$ is the dilation operator defined by: $\left(D_{\lambda} g\right)(x)=g\left(\lambda^{-1} x\right)$. Let $B_{l}(x)$ be the "box" centered at $x=(u, v)$ having size l: $B_{l}(x)=\left[u-\frac{l}{2}, u+\frac{l}{2}\right] \times\left[v-\frac{l}{2}, v+\frac{l}{2}\right]$.

Definition 1. An image descriptor $\mathbf{q}: G \rightarrow \mathbf{R}^{n}$ is a real functional which depends only on the image inside $B_{1}(0): \mathbf{q} \cdot g=\mathbf{q} \cdot g I_{B_{1}(0)}$ (We use the dot product notation to denote the operation of applying an image descriptor to an image, i.e. $\mathbf{q} \cdot g=\mathbf{q}(g)) . I_{B_{1}(0)}$ is the characteristic function of $B_{1}(0): I_{B_{1}(0)}(x)=1$ for $x \in B_{1}(0)$ and $I_{B_{1}(0)}(x)=0$ for $x \notin B_{1}(0)$.

Definition 2. The image description $\tilde{\mathbf{g}}(x)$ generated by $\mathbf{q}$ is defined by: $\tilde{\mathbf{g}}(x)=\mathbf{q} \cdot T_{-x} g$. For each component we have then $\tilde{g}_{i}(x)=q_{i} \cdot T_{-x} g$.

The definitions and results below are valid for any image descriptor "regular" enough (see [10] for a precise definition). Most of the texture features used in the literature can be adapted to fit into this formalism. However, to give an example (and because we use it in our implementation) we discuss a particular case in more detail. Namely, given a set functions $h_{i}, i=1, \ldots, n$ such that $h_{i}(x)=0$ for $x \notin B_{1}(0)$, consider the following descriptor:

$$
q_{i} \cdot g=f_{i}\left[\int h_{i}\left(x^{\prime}\right) g\left(-x^{\prime}\right) \mathrm{d} x^{\prime}\right] .
$$


The purpose of the function $f_{i}: \mathbf{R} \rightarrow \mathbf{R}$ is to introduce a non-linearity. The image description $\tilde{\mathbf{g}}(x)=\mathbf{q} \cdot T_{-x} g$ is then obtain from $g$ by a convolution cascaded with the non-linearity $f_{i}: \tilde{g}_{i}(x)=q_{i} \cdot T_{-x} g=f_{i}\left[\int h_{i}\left(x^{\prime}\right) g\left(x-x^{\prime}\right) \mathrm{d} x^{\prime}\right]=f_{i}\left[\left(h_{i} * g\right)(x)\right]$.

\subsection{Homogeneous Random Images.}

Let $(\Omega, \mathcal{F}, \mathcal{P})$ be a probability space. A random image $\psi$ is a function defined on $\Omega$ into the set of images: $\psi: \Omega \rightarrow G$. That is, if $\omega \in \Omega$, then $\psi(\omega) \in G$ is an image.

Definition 3. A random image $\psi$ is homogeneous if for any image descriptor $\mathbf{q}$, for all $x_{0} \in \mathbf{R}^{2}$ and for almost all $\omega \in \Omega$ :

$$
\lim _{l \rightarrow \infty} \frac{1}{l^{2}} \int_{B_{l}\left(x_{0}\right)} \mathbf{q} \cdot T_{-x} \psi(\omega) \mathrm{d} x=\Psi_{\mathbf{q}}
$$

where $\Psi_{\mathbf{q}} \in \mathbf{R}^{n}$ is the asymptotic description of the random homogeneous image $\psi$ given by the descriptor $\mathbf{q}$.

To clarify the meaning of (2) let us rewrite it for the convolution-plus-nonlinearity case defined in (1): $\lim _{l \rightarrow \infty} \frac{1}{l^{2}} \int_{B_{l}\left(x_{0}\right)} q_{i} \cdot T_{-x} \psi(\omega) \mathrm{d} x=\lim _{l \rightarrow \infty} \frac{1}{l^{2}} \int_{B_{l}\left(x_{0}\right)} f_{i}\left[\left(h_{i} * \psi(\omega)\right)(x)\right] \mathrm{d} x=$ $\lim _{l \rightarrow \infty} \frac{1}{l^{2}} \int_{B_{l}\left(x_{0}\right)} \tilde{g}_{i}(x) \mathrm{d} x=\Psi_{\mathbf{q}, i}$ where $\Psi_{\mathbf{q}}=\left(\Psi_{\mathbf{q}, i}: i=1, \ldots, n\right)$ and $\tilde{g}_{i}(x)$ is given by: $\tilde{g}_{i}(x)=f_{i}\left[\left(h_{i} * \psi(\omega)\right)(x)\right]$. That is, homogeneity requires that averages of image descriptions exist in the thermodynamical limit and be spatial independent.

This definition is quite general. It includes all ergodic random functions, in which case $\Psi_{\mathbf{q}}$ is the ensemble average of the image description. It is also valid for periodic deterministic functions such as those generated by regular repetitive texture.

However, if illumination inhomogeneities or distorsions such as those created by prospective are present, then our model of texture is no longer valid at very large scales. A more complex model is needed in which our homogeneous random function is coupled with (perturbed by) a smooth, slowly varying function. If this perturbation is small enough it is still possible to approach the thermodynamic limit before the long range variations become important. The greatest degree of homogeneity is then attained at a finite statistical scale.

\subsection{Multiscale Representations.}

The image description $\tilde{\mathbf{g}}(x)=\mathbf{q} \cdot T_{-x} g$ is obtained by applying the descriptor $\mathbf{q}$ to the translated image. However, translation is not the only natural syminetry of images. Another one is dilation ${ }^{5}$. We are then led to the following definition:

Definition 4. The multiscale description of $g \in G$ given by the descriptor $q$ is the function: $\hat{\mathbf{g}}: \mathbf{R}^{+} \times \mathbf{R}^{2} \rightarrow \mathbf{R}^{n}$ given by:

$$
\hat{g}_{i}(\lambda, x)=K_{i}(\lambda)\left(q_{i} \cdot D_{\lambda^{-1}} T_{-x} g\right) .
$$

where $K_{i}(\lambda)$ is a normalization factor.

${ }^{5}$ Rotation is also a natural symmetry. However, since this paper focuses on scale issues we omit to deal with orientation explicitly. 
For the convolution-plus-nonlinearity descriptor defined in (1) we have, after some simple calculation: $\hat{g}_{i}(\lambda, x)=K_{i}(\lambda) f_{i}\left[\left(\left(D_{\lambda} h_{i}\right) * g\right)(x)\right]$. In this case, the i-th component of the multiscale description is obtained by convolving the image with a bank of filters generated by dilating the template filter $h_{i}$. Wavelets representations can be expressed in this way by letting $\lambda=2^{k}$ and $x=\left(l 2^{k}, m 2^{k}\right), l, m, k \in \mathbf{Z}$ and choosing $h_{i}, f_{i}$ in the appropriate way.

Note that $\hat{\mathbf{g}}(\lambda, x)$ depends only on the image inside $B_{\lambda}(x)$.

\subsection{Averaged Multiscale Representations.}

Definition 5. The averaged multiscale description of $g \in G$ given by the descriptor $\mathbf{q}$ is the function: $\overline{\mathbf{g}}: \mathbf{R}^{+} \times \mathbf{R}^{+} \times \mathbf{R}^{2} \rightarrow \mathbf{R}^{n}$ given by, for $\lambda<L$ :

$$
\overline{\mathbf{g}}(L, \lambda, x)=\frac{1}{(L-\lambda)^{2}} \int_{B_{L-\lambda}(x)} \hat{\mathbf{g}}\left(\lambda, x^{\prime}\right) \mathrm{d} x^{\prime} .
$$

For $\lambda \geq L$ we let $\overline{\mathbf{g}}(L, \lambda, x)=0$.

Note that the average is taken in such a way that $\overline{\mathbf{g}}(L, \lambda, x)$ depends only on the image inside $B_{L}(x)$.

If $g=\psi(\omega)$ is a homogeneous random image then, by definition of homogeneity (see (2)), we have with probability 1 and for all $x \in \mathbf{R}^{2}$ (assuming $K_{i}(\lambda)=K(\lambda)$ for clarity):

$$
\lim _{L \rightarrow \infty} \overline{\psi(\omega)}(L, \lambda, x)=\lim _{L \rightarrow \infty} \frac{1}{(L-\lambda)^{2}} K(\lambda) \int_{B_{L-\lambda}(x)} \mathbf{q} \cdot D_{\lambda^{-1}} T_{-x^{\prime}} \psi(\omega) \mathrm{d} x^{\prime}=\Psi(\lambda)
$$

Definition 6. The function $\Psi: \mathbf{R}^{+} \rightarrow \mathbf{R}^{n}$ will be called multiscale asymptotic description of $g$.

\subsection{Definition of the dishomogeneity function.}

We are going to define a dishomogeneity function $g^{\prime}(L, x) \geq 0$ such that $g^{\prime}(L, x)$ depends only on the image inside $B_{2 L}(x)$ and on averages of size $L$. We start by defining the maximum and minimum value of $\bar{g}_{i}(L, \lambda, x)$ inside $B_{2 L}(x)$ :

$$
\bar{g}_{i}^{\mathbf{M}}(L, \lambda, x)=\max _{x^{\prime} \in B_{L}(x)} \bar{g}_{i}\left(L, \lambda, x^{\prime}\right) \quad \bar{g}_{i}^{\mathrm{m}}(L, \lambda, x)=\min _{x^{\prime} \in B_{L}(x)} \bar{g}_{i}\left(L, \lambda, x^{\prime}\right)
$$

Now, let $s_{i}(M, m)$ be a positive real function defined for $M \geq m \geq 0$ such that $s_{i}(M, m)=0$ if $M=m$ and, for each $m, s_{i}(M, m)$ is strictly increasing in $M$ (the simplest example would be $\left.s_{i}(M, m)=M-m\right)$.

Definition 7. The specific dishomogeneity is: $g_{i}^{\prime}(L, \lambda, x)=s_{i}\left(\bar{g}_{i}^{\mathrm{M}}(L, \lambda, x), \bar{g}_{i}^{\mathrm{m}}(L, \lambda, x)\right)$.

Definition 8. The dishomogeneity function is then defined by taking the most dishomogeneous "channel":

$$
g^{\prime}(L, x)=\max _{i, \lambda} g_{i}^{\prime}(L, \lambda, x)
$$




\subsection{Asymptotic properties of the dishomogeneity function.}

A first property of the dishomogeneity function is the following (for proofs see [10]):

Theorem 9. Let $\psi(\omega)$ be a homogeneous random image and let $[\psi(\omega)]^{\prime}(L, x)$ be its dishomogeneity function. Then $\lim _{L \rightarrow \infty}[\psi(\omega)]^{\prime}(L, x)=0$ for all $x \in \mathbf{R}^{2}$ and with probability 1 .

To deal with images which contain more than one type of texture we need a model for them. A simple one is a piecewise homogeneous random function. Let then $P=$ $\left\{R_{1}, \ldots, R_{n}\right\}$ be a partition of $\mathbf{R}^{2}: R_{i} \subset \mathbf{R}^{2} ; R_{i} \cap R_{j}=\emptyset$ if $i \neq j ; \bigcup R_{i}=\mathbf{R}^{2}$. We assume that boundaries are regular enough, for instance, piecewise smooth. Let $\left\{\psi_{i}: i=1, \ldots, n\right\}$ be a set of homogeneous random images.

Definition 10. A piecewise homogeneous random image over the partition $P$ is the random function: $\phi=\sum_{i=1}^{n} I_{R_{i}} \psi_{i}(\omega)$.

Definition 11. We say that $\phi$ is asymptotically discriminable (by the image descriptor $\mathbf{q}$, used to define the dishomogeneity function) if all $\psi_{i}, i=1, \ldots, n$ have different asymptotic descriptions, i.e. $\Psi_{i} \neq \Psi_{j}$ for $i \neq j$.

Then we have to define what the thermodynamic limit means for these class of images. For, it is no longer possible to let $L$ go to infinity without mixing together different types of texture. A solution to this problem is to make the random functions $\psi_{i}$ "shrink" while leaving the boundaries unchanged. Then, for $\phi$ defined as above and $\gamma>0$ we define $\phi_{\gamma}$ as: $\left[\phi_{\gamma}(\omega)\right](x)=\sum_{i=1}^{n} I_{R_{i}}(x)\left[\psi_{i}(\omega)\right](\gamma x)$.

Definition 12. For each $\omega \in \Omega$ the asymptotic dishomogeneity function of $\phi(\omega), \phi_{\infty}^{\prime}(\omega)$ : $\mathbf{R}^{+} \times \mathbf{R}^{2} \rightarrow \mathbf{R}^{+}$, is given by: $\left[\phi_{\infty}^{\prime}(\omega)\right](L, x)=\lim _{\gamma \rightarrow \infty}\left[\phi_{\gamma}(\omega)\right]^{\prime}(L, x)$.

Theorem 13. With the above definitions we have with probability 1:

1) The above limit exists for all $\boldsymbol{x} \in \mathbf{R}^{2}, L>0$

2) If $B_{2 L}(x) \subset R_{i}$ for some $i$ then $\left[\phi_{\infty}^{\prime}(\omega)\right](L, x)=0$

3) If $\phi$ is asymptotically discriminable, and if $B_{2 L}(x)$ contains at least two different types of texture, i.e. $B_{2 L}(x) \cap R_{i} \neq \emptyset, B_{2 L}(x) \cap R_{j} \neq \emptyset, i \neq j$, then $\left[\phi_{\infty}^{\prime}(\omega)\right](L, x)>0$

This theorem suggests that in finite images the dishomogeneity function can provide useful information wherever the thermodynamic limit is a reasonable approximation.

\section{Finding Texture Boundaries.}

In real images the thermodynamic limit is at best an ideal approximation. Moreover, it can not be attained near boundaries with high curvature, even if all homogeneous regions are very large. Nonetheless, the dishomogeneity function can be very useful for detecting the statistical scale at which relevant events - namely, the presence of a homogeneous texture - occur. This can be done at each point $x$ in the image by looking for minima of the dishomogeneity function $g^{\prime}(L, x)$ with respect to $L . g^{\prime}(L, x)$ can also be useful to find boundaries, since we expect abrupt increases of $g^{\prime}(L, x)$ when the corresponding window, $B_{2 L}(x)$, "invades" a nearby texture.

A complete description of our algorithm can be found in [11]. Here we just give a brief sketch of it. The algorithm is embedded in an overlapped pyramid of the type shown in figure 4 . Each node in the pyramid corresponds to a square window. A cost 
$c_{k i j}=g_{k i j}^{\prime}-\alpha t_{k}$ is associated with each node. $t_{k}$ is increasing in $k$. The negative term $-\alpha t_{k}$ introduces a bias for large statistical scales. This allows to select a unique scale and to generate a consistent segmentation in those cases where the underlying texture is near the thermodynamic limit at more than one statistical scale (for instance, think of a checkerboard). The dishomogeneity is computed as described in section 2 by using filter-plus-nonlinearity descriptor of the type shown in (1). Step filters at 4 different orientations have been used.

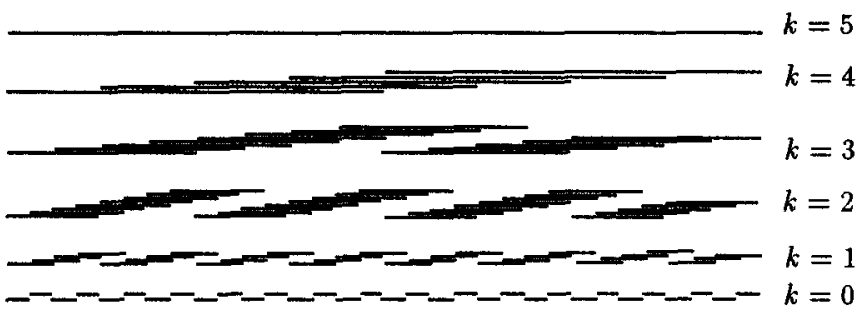

Fig. 4. One dimensional oversampled pyramid. The vertical displacement between intervals of the same level has been introduced for clarity and has no other meaning. Each node of the pyramid has a dishomogeneity value and a cost associated with it.

The algorithm is region based, i.e. its output is a partition of the image. Therefore it assumes that boundaries form closed curves and are sharp enough everywhere. It works in two steps: first it selects nodes in the pyramid which minimize locally the cost function $c_{k i j}$ and then merges neighboring selected nodes into larger regions. In the selection phase each pixel of the image selects a unique node (the one having the lowest cost) among all those in which it is contained.

\section{Experiments}

We now describe some of the experiments we have done with synthetic images. All the three images shown in this section are $256 \times 256$. The CPU time required to run one image is approximately 9 minutes on a Sun SparcStation II. Most of the time goes into the computation of $\bar{g}_{i}^{\mathrm{M}}(L, \lambda, x)$ and $\bar{g}_{i}^{\mathrm{m}}(L, \lambda, x)$ from $\bar{g}_{i}\left(L, \lambda, x^{\prime}\right)$ (see section 2.5$)$.

Figure 5 shows the segmentation of a collage of textures which reach the thermodynamic limit at several statistical scales.

Figure 6-left illustrates the segmentation of an "order versus disorder" image. This example shows that looking for the optimal statistical scale can significantly enhance discriminative capabilities making possible the detection of very subtle differences.

Finally, figure 6-right shows that this scheme can also be valid for textures whose properties change smoothly across the image (as occurs when tilt or slant are present).

\section{Conclusions}

In this paper we have addressed the problem of scale selection in texture analysis. We have proposed to make a clear distinction between two different scale parameters: statistical scale and feature scale. Both scale parameters should be taken into account in 

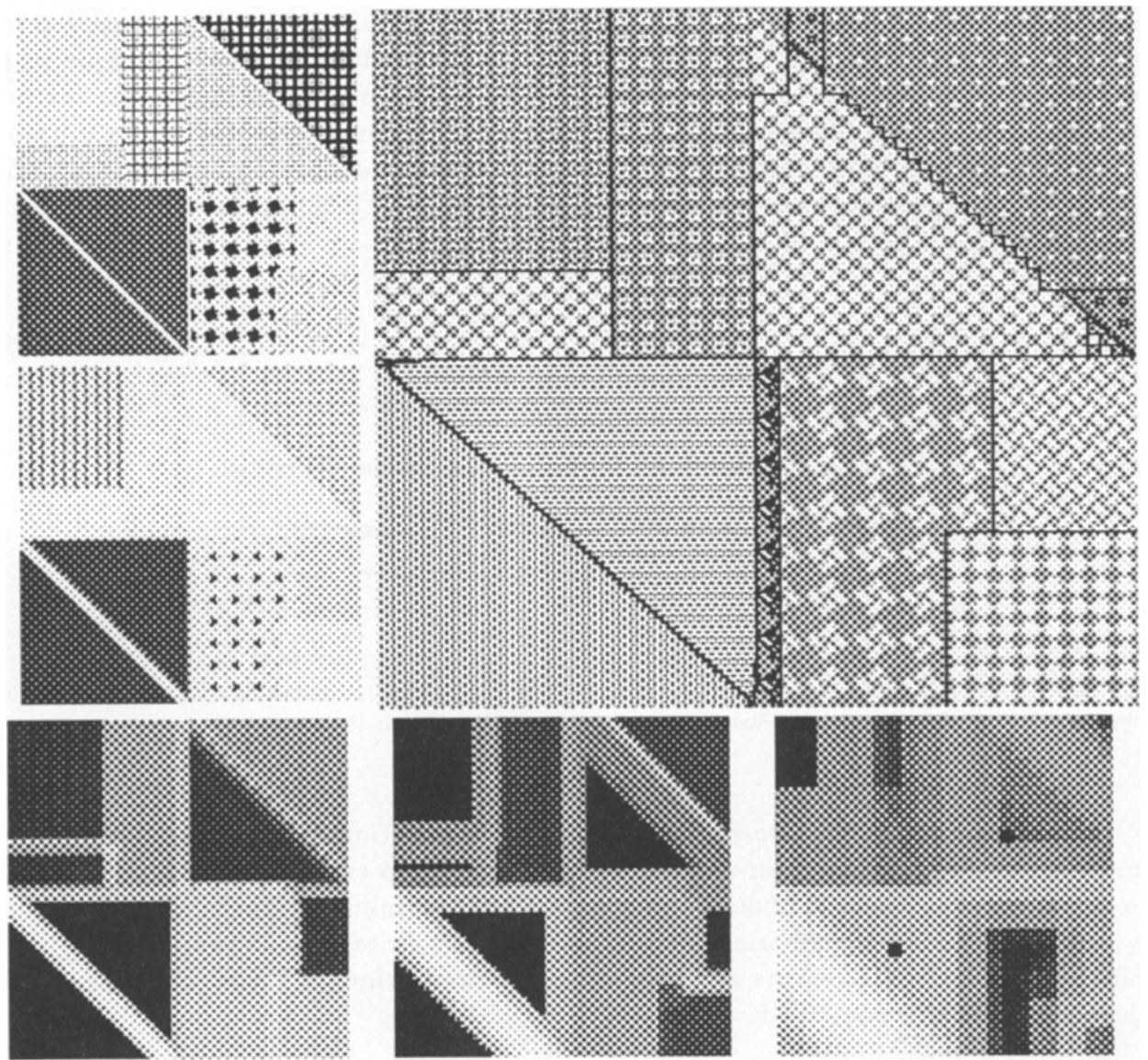

Fig. 5. Top-right: a $256 \times 256$ textured image. The black lines are the boundaries found by the algorithm. Left and bottom: the dishomogeneity $g^{\prime}(L, x)$ for $L=2^{k}, k=1, \ldots, 5$. $k$ grows anti-clockwise. Homogeneous regions are black. Note that the thermodynamic limit is attained at different statistical scales by different textures.
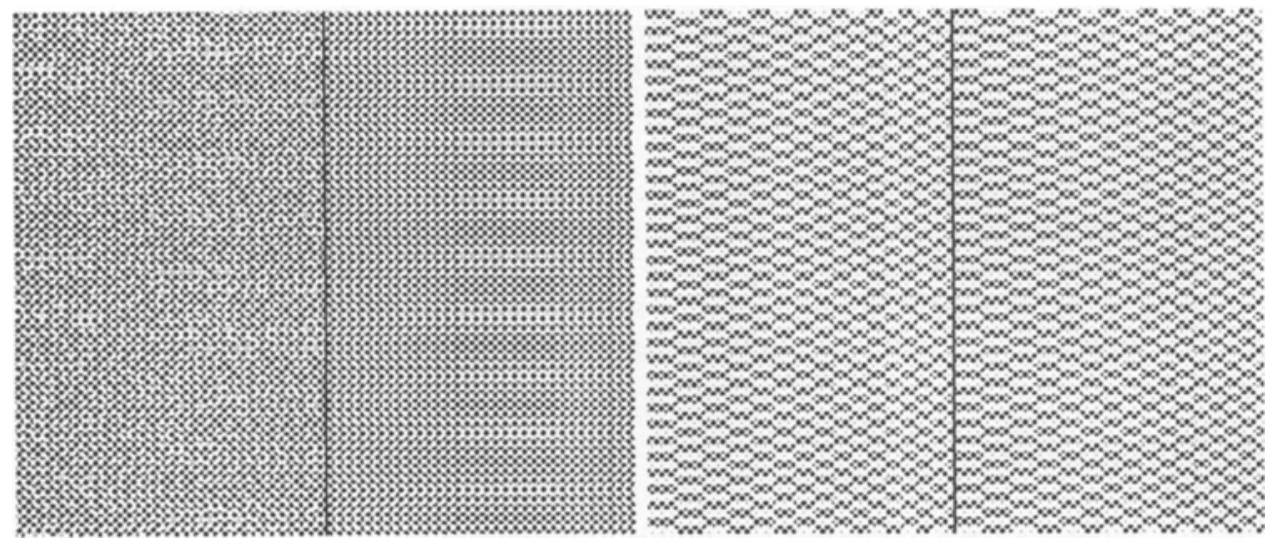

Fig. 6. Two $256 \times 256$ textured images: "Order versus disorder" and "tilted texture". 
constructing image representations but they should be dealt with in very different ways. In particular, we claim that it is necessary to find the optimal statistical scale(s) at each location in the image. In doing this there is a natural trade-off between the reliable estimation of image properties and the localization of texture regions. It is possible to extract texture boundaries reliably only if a good enough trade-off can be found.

We have formalized the notion of homogeneity by the definition of homogeneous random functions. When local operators are applied to these functions and the result is averaged over regions of increasing size, we obtain a description of the image which is asymptotically deterministic and space independent. In practical circumstances, we say that the thermodynamic limit has been reached when this holds to a sufficient degree. We have defined a dishomogeneity function and proved that in the thermodynamic limit it is zero if and only if the analyzed region does not contain a texture boundary.

Our algorithm has performed well on images which satisfy the piecewise-homogeneous assumption. However, it did not perform well on images which violate the piecewise homogeneous property, mainly because in such images boundaries are not sharp enough everywhere and are not well defined closed curves. Our node-merging phase is not robust with respect to this problem. We are currently designing an algorithm which is more edge-based and should be able to deal with boundaries which are not closed curves. Also, we need to use a better set of filters.

\section{References}

1. H. Knuttson and G. H. Granlund. Texture analysis using two-dimensional quadrature filters. In Workshop on Computer Architecture for Pattern Analysis ans Image Database Management, pages 206-213. IEEE Computer Society, 1983.

2. M.R. Turner. Texture discrimination by gabor functions. Biol. Cybern., 55:71-82, 1986.

3. J. Malik and P. Perona. Preattentive texture discrimination with early vision mechanisms. Journal of the Optical Society of America - A, 7(5):923-932, 1990.

4. A.C. Bovik, M. Clark, and W.S. Geisler. Multichannel texture analysis using localized spatial filters. IEEE Trans. Pattern Anal. Machine Intell., 12(1):55-73, 1990.

5. B. Julesz. Visual pattern discrimination. IRE Transactions on Information Theory IT-8, pages 84-92, 1962.

6. R. L. Kashyap and K. Eom. Texture boundary detection based on the long correlation model. IEEE transactions on Pattern Analysis and Machine Intelligence, 11:58-67, 1989.

7. D. Geman, S. Geman, C. Graffigne, and P. Dong. Boundary detection by constraint optimization. IEEE Trans. Pattern Anal. Machine Intell., 12(7):609, 1990.

8. R. Wilson and G.H. Granlund. The uncertainty principle in image processing. IEEE Trans. Pattern Anal. Machine Intell., 6(6):758-767, Nov. 1984.

9. M. Spann and R. Wilson. A quad-tree approach to image segmentation which combines statistical and spatial information. Pattern Recogn., 18:257-269, 1985.

10. S. Casadei. Multiscale image segmentation by dishomogeneity evaluation and local optimization (master thesis). Master's thesis, MIT, Cambridge, MA, May 1991.

11. S. Casadei, S. Mitter, and P. Perona. Boundary detection in piecewise homogeneous textured images (to appear). Technical Report -, MIT, Cambridge, MA, - -. 